\title{
Treatment with Tyrosine Kinase Inhibitors in Chronic Myeloproliferative Neoplasms: Pros and Cons
}

\author{
Safaa A. A. Khaled \\ Department of Internal Medicine, Hematology \& BMT Unit, Assiut University Hospital, Faculty of Medicine, Assiut University, Assiut, \\ Egypt
}

\section{Email address:}

sa_ah_mh@yahoo.com, safaakhaled2003@gmail.com

\section{To cite this article:}

Safaa A. A. Khaled. Treatment with Tyrosine Kinase Inhibitors in Chronic Myeloproliferative Neoplasms: Pros and Cons. American Journal of Internal Medicine. Vol. 4, No. 4, 2016, pp. 66-74. doi: 10.11648/j.ajim.20160404.12

Received: June 10, 2016; Accepted: June 20, 2016; Published: July 4, 2016

\begin{abstract}
Myeloproliferative neoplasms (MPNs) are a group of hematological disorders characterized by proliferation of one or more than one myeloid lineage. Genetically they express single or multiple mutations of the Janus tyrosine kinase receptors. Advances in understanding molecular and cytogenetic pathophysiology of MPNs led to further identification of different mutations rather than the classical break point cluster region Abelson (BCR-ABL). Although the onset of disease in all MPNs is insidious and may be asymptomatic, also MPNs run a slowly progressive course however they carry the potential of blastic transformation. Furthermore, peripheral blood leucocytosis, thrombocytosis or erythroctosis can lead to a wide array of fatal complications. Originally treatment of MPNs based on cytoreduction and supportive measures. Tyrosine kinase inhibitors (TKIs) are a group of anti-neoplastic drugs that specifically targeting malignant cells. Many studies proved the efficacy and safety of TKIs in management of patients with MPNs. This study was conducted to evaluate TKIs, pros and cons.
\end{abstract}

Keywords: MPNs, Pros and Cons, TKIs

\section{Introduction}

MPNs are the 2008 WHO nomenclature of the myeloproliferative disorders (MPDs). They included the classical MPDs, chronic myeloid leukemia (CML), primary myelofiberosis (PMF), polycythemia vera (PV) and essential thrombocythemia (ET), together with chronic eosinophilic leukemia (EL), systemic mastocytosis and unclassifiable MPNs. Furthermore, in 2008 WHO classification of myeloid/lymphoid neoplasms CML was categorized into BCR-ABL positive CML that was included with MPN and BCR-ABL negative CML that was classified with MDN/MPN. [1]

MPNs are a group of clonal stem cell disorders that share some clinical features, at the molecular level they are characterized by presence of mutations at the gene coding the tyrosine kinase family. An activating mutation of the BCRABL gene leading to active JAk-1 was detected in CML patients, whereas a substitution of phenylalanine with valine at position 617 at JAk-2 gene was found in patients with PV, PMF and ET. [2-4] Epidemiologically the most common
MPN is CML, epidemiological studies of other types were insufficient, however all MPNs are uncommon below 20years and rare in childhood. They affect all racial groups with a slight male predominance, male to female ratio 1.4:1. [5, 6]

A breakthrough in management of MPNs occurred in the start of the twentieth century with the introduction of the targeted effective therapies TKIs. The BCR- ABL specific tyrosine kinase inhibitor, imatinib (IM) mesylate was found to induce complete cytogenetic response (CCyR) in $75 \%$ of CML patients by 2-years, major molecular response (MMR) in $50 \%$ after 5 -years and complete molecular response in $10 \%$. [7, 8] Imatinib mesylate is a first generation TKI, dasatinib and nilotinib are second generation TKIs that were recommended for CML patients with IM failure or resistance. [9] In 2011, the US food and drug administration (FDA) approved the multi- TKI, Ruxolitinib for patients with myelofiberosis and for PV. $[10,11]$

Adherence to medical treatment was found very critical for achieving proper therapeutic response in any given disease. There are various methods to measure patients' adherence to treatment particularly the orally administered ones. [12, 13] Treatment of MPNs is similar to treatment of any chronic 
disorder is subjective to be influenced by non-adherence. [14]

This study was conducted to assess advantages and disadvantages of TKIs in MPNs, in terms of accessibility, affordability, tolerability, therapeutic response, patients' adherence and outcome of patients.

\section{Patients and Methods}

\subsection{Study Patients and Data Collection}

Patients were recruited from those who were attending the hematology outpatient clinic for follow up (a visit every 2weeks) or admitted at the Hematology Unit, Internal Medicine Department, Assiut University Hospital (AUH), over a period of 8-months. Prior diagnosis of MPN was an inclusion criterion while those with newly diagnosed MPN were excluded from the study. Patients who received TKIs as first or second line treatment were included in the study, also those who received $\mathrm{HU}$ or other drugs were enrolled for comparison.

Demographic and clinical data were collected through direct patient interview, thorough history and clinical examination. CBC, ESR, NAP score, bone marrow biopsy or aspirate (if indicated), and cytogenetic analyses were done for each participant as a part of their follow up investigations.

Diagnosis of MPNs in the study group was based on guidelines for diagnosis of MPNs, [15-18] confirmed by reviewing patients' data on their first visit. Phases of CML were diagnosed according to Kantarjian et al. [19]

\subsection{Treatment of the Study Participants}

According to previously published guidelines by others, [20] patients with chronic phase or accelerated phase CML received IM 400-mg/once daily. This dose was doubled in cases of failure to obtain response, and reduced to $300-\mathrm{mg}$ in presence of side effects. Those who developed IM failure were managed with dasatinib 100-mg once daily or HU. A group of patients were treated with HU as first line treatment; the starting dose was 500-mg twice daily that was titrated according to TLC.

Asymptomatic patients with PMF did not receive any treatments; symptomatic patients were managed with supportive measures $\pm \mathrm{HU} \pm$ splenic irradiation. [21] PV was treated with phlebotomy, low dose aspirin and HU. [22] However Ruxolitinib was not available for our patients. ET was treated with low dose aspirin, HU and platelet pharesis (in severe cases). EL was treated with corticosteriods and hydroxyura. [23]

\subsection{Assesment of Incompliance to Medications in the Study Group}

Incompliance to medications was measured by self-reporting method in which patients were inquired about adherence to medications and factors of non-adherence. The later were grouped into 5- categories patient, disease, health system, social and economic, and treatment centered factors. [24]

\subsection{Definitions and Assessment of Response to Treatment in the Study Participants}

For patients with CML complete hematologic response (CHR) was defined as asymptomatic disease with impalpable spleen, the C.B.C showed TLC $<10 \times 10^{9} / 1$, with normal DLC, platelet count $<450 \times 10^{9} / 1$, and $<1 \%$ immature cells in the peripheral blood. Partial response (PHR) was considered if patient is asymptomatic, or with palpable splenomegaly, or TLC $10-20 \times 10^{9} / 1$, or $>1 \%$ immature cells in presence of normal C.B.C. Treatment failure was included primary resistance with failure to obtain CHR after 3-months and secondary resistance with loss of a previously obtained therapeutic response. Progressive disease was defined as acceleration or blastic transformation. [25]

For BCR-ABL negative MPNs we followed guidelines recommended by others to assess treatment responses. [23]

PFS was defined as the time from diagnosis of disease to progression, and OS as the time from diagnosis to death or last follow up. [25]

\subsection{Ethical Considerations}

The study design, and methodology were consistent with the World Medical Association (WMA) declaration of Helsinki for ethics in medical research. [26] Data collection was approved by the research ethical committee of faculty of Medicine, AUH. Research protocol and objectives were discussed to all the study participants before enrollment in the study. Patients' consent for participation in the study was mandatory. Furthermore all patients knew well that they can withdraw from the study at any time without affecting their medical care.

\subsection{Statistical Analysis}

Data were collected then introduced into a personal computer substituting patients' names with code numbers. Analysis of the collected data was done with SPSS V. 17 software (SPSS Inc. Chicago, TL, USA), and Graph Pad Prism V5, Italy. The mean \pm SD, median, and range were used to describe quantitative variables while qualitative variables were expressed as percentages from the total number. The $X^{2}$ test was used to analyze differences among qualitative variables. The Tukey's multiple comparison tests was used to estimate differences between study patients who were grouped according to treatment into 3-groups, those who received TKIs as first, TKIs as second and HU as first or second line treatment.

\section{Results}

\subsection{Demographic, Clinical and Disease Characteristics of the Study Participants}

47 patients with MPNs were included in the study, their median age was 50-years and range between $18-80$ years old, $59.6 \%$ were females and the male to female ratio was $1: 1.4$. Most of patients were from Assiut Governorate (72.3\%) and 
$51 \%$ were from rural community, table 1 showed demographic characteristics of the study group.

$55.3 \%, 57.4 \%, 85.1 \%$ of patients presented with abdominal pain, huge splenomegaly and hepatomegaly, respectively. ECOG PS was fully active in $66 \%$. Median TLC was $71 \times 10^{3} / \mathrm{mm}^{3} .55 .3 \%$ of patients were Philadelphia positive, and table 2 showed clinical and disease characteristics of the study group.

CML comprised $78.7 \%$ of patients in the study group, and 7 patients with CML \& 2 with PMF progressed to blastic transformation. One patient with PV developed fibrosis, and figure 1 demonstrated distribution of MPNs among the study participants.

$25.5 \%$ of patients received TKIs (IM) as first line treatment and $27.7 \%$ as second line, of the later $21.3 \%$ received IM secondary to $\mathrm{HU}$ and $6.4 \%$ dasatinib secondary to IM. 19 patients were treated with HU, in 17 of them HU was first line drug and in 2 of them it was second line to IM. $6.3 \%$ of the study group were treated with other drugs, as corticosteroids in EL. $55.7 \%$ of patients were non-compliant to medication; the median duration of non-compliance was 12-days, table 2.

Table 1. Demographic characteristics of the study participants ( $n=47)$.

\begin{tabular}{l|l}
\hline Variable & Estimated results \\
\hline Gender & $19(40.4 \%)$ \\
Male & $28(59.6 \%)$ \\
Female & \\
Age & $18-80$ \\
Range & $46.17 \pm 16.03$ \\
Mean \pm SD & 50 \\
Median & \\
Residence & $34(72.3 \%)$ \\
Assiut & $3(6.4 \%)$ \\
Sohag & $4(8.5 \%)$ \\
Qena & $2(4.3 \%)$ \\
Luxor & $2(4.3 \%)$ \\
Aswan & $2(4.3 \%)$ \\
Al-Menia & \\
Social environment & $23(48.9 \%)$ \\
Urban & $24(51.1 \%)$ \\
Rural & \\
Occupation & $22(46.8 \%)$ \\
\hline Housewife & $5(10.6 \%)$ \\
Employed & $9(19.1 \%)$ \\
Farmer & $8(17.0 \%)$ \\
\hline Snemployed & $3(6.4 \%)$ \\
\hline Student & \\
\hline & \\
\hline
\end{tabular}

N.B. $\mathrm{SD}=$ standard deviation.

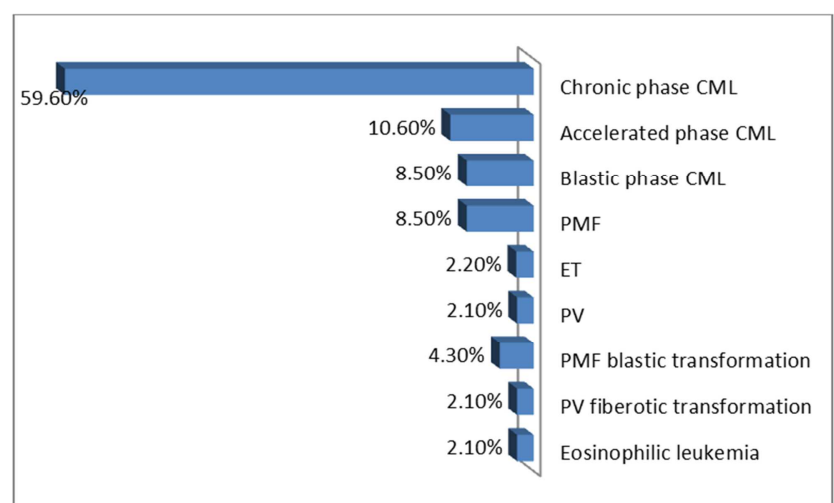

Figure 1. Distribution of myeloproliferative neoplasms in the study group $(n=47)$. N.B. $C M L=$ chronic myeloid leukemia, $M F=$ primary myelofiberosis, $P V=$ polycythemia vera, ET= essential thrombocythemia.

Table 2. Clinical, disease and treatment characteristics of the study participants $(n=47)$.

\begin{tabular}{|c|c|}
\hline Variable & Frequency $(\%)$ \\
\hline \multicolumn{2}{|l|}{ Clinical characteristics } \\
\hline \multicolumn{2}{|l|}{ Complaint: } \\
\hline Abdominal pain & $26(55.3 \%)$ \\
\hline Manifestations of anemia & $18(38.3 \%)$ \\
\hline Manifestations of pancytopenia & $2(4.3 \%)$ \\
\hline Atypical chest pain & $1(2.1 \%)$ \\
\hline \multicolumn{2}{|l|}{ ECOG PS } \\
\hline Fully active & $31(66.0 \%)$ \\
\hline Restricted strenuous activity & $18(27.6 \%)$ \\
\hline Self-care but no work & $3(6.4 \%)$ \\
\hline \multicolumn{2}{|l|}{ Spleen } \\
\hline Huge splenomegaly & $27(57.4 \%)$ \\
\hline Mild to moderate & $20(42.6 \%)$ \\
\hline \multicolumn{2}{|l|}{ TLC } \\
\hline Range & $3-546$ \\
\hline Mean \pm SD & $150.22 \pm 159.20$ \\
\hline Median & 71 \\
\hline \multicolumn{2}{|l|}{ Liver } \\
\hline Hepatomegaly & $40(85.1 \%)$ \\
\hline Impalpable & $7(14.9 \%)$ \\
\hline \multicolumn{2}{|l|}{ Ph chromosome } \\
\hline Positive & $26(55.3 \%)$ \\
\hline Negative & $21(44.7 \%)$ \\
\hline \multicolumn{2}{|l|}{ Treatment } \\
\hline TKIs (first line) & $12(25.5 \%)$ \\
\hline HU (first or second line) & $19(40.5 \%)$ \\
\hline TKIs (second line) & $13(27.7 \%)$ \\
\hline Others & $3(6.3 \%)$ \\
\hline \multicolumn{2}{|l|}{ Patient Compliance } \\
\hline -Compliant & $21(47.3 \%)$ \\
\hline -Non-Compliant & $26(55.7 \%)$ \\
\hline \multicolumn{2}{|c|}{ Duration of non-compliance in days } \\
\hline Range & $10-60$ \\
\hline Median & 12 \\
\hline Mean \pm SD & $46.17 \pm 16.03$ \\
\hline
\end{tabular}

N.B. ECOG PS= Eastern co-operative group performance status, $\mathrm{MPN}=$ Myeloproliferative neoplasms, $\mathrm{CML}=\mathrm{Chronic}$ myeloid leukemia, $\mathrm{PMF}=$ Primary myelofiberosis, $\mathrm{ET}=$ Essential thrombocythemia, $\mathrm{PV}=$ Polycythemia vera, $\mathrm{EL}=$ Eosinophilic leukemia, $\mathrm{Ph}=$ Philadelphia, TKIs=tyrosine kinase inhibitors, $\mathrm{HU}=$ Hydroxyurea. 


\subsection{Drug Side Effects and Factors of Non-compliance in Patients Treated with TKIs Compared to Other Drugs}

Non-hematological side effects of medications such as raised ALT\& AST, edema of lower limbs and periorbital puffiness, and portal vein thrombosis were only found in patients who received TKIs as second line treatment. Nausea and vomiting were mainly encountered in those who received HU (55.4\%) followed by TKIs second line (46.6\%) and lastly TKIs first line.

Hematological side effects, such as thrombocytopenia \&leucopenia were present only in those who received TKIs as first \& second line drugs, respectively. Pancytopenia was present in similar proportion (44.4\%) of patients who received HU and TKIs second line. Anemia was found in $46.2 \%$, $30.8 \%$, and $23.1 \%$ of patients who received TKIs first line, $\mathrm{HU}$, and TKIs second line respectively, as depicted in figure 2.

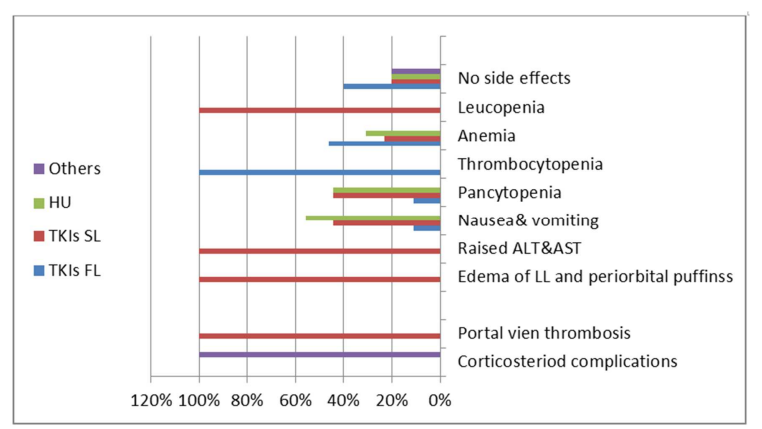

Figure 2. Hematologic and non-hematologic side effects of TKIs compared to other drugs among the study group, $P=0.002$. N.B. TKIs=tyrosine kinase inhibitors, $H U=$ hydroxyurea, $F L=$ first line drug, $S L=$ second line drug.

Factors of non- compliance in patients who received TKIs as first line treatment were pregnancy, absent symptoms, cost, lack of accessibility, and side effects in order of frequency. The most common factors for those who received TKIs second line and HU were lack of accessibility and fluctuated course of disease, respectively, as in figure 3.

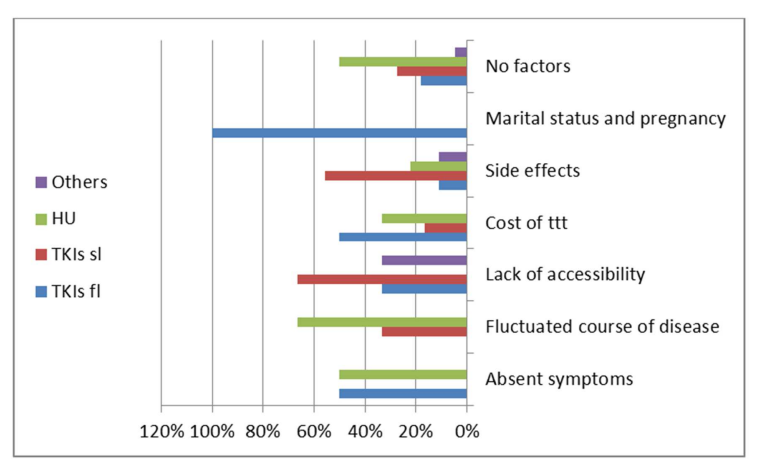

Figure 3. Factors of non-compliance among patients who were treated with TKIs compared to other drugs, $P=0.310$. N.B. TKIs $=$ tyrosine kinase inhibitors, $H U=$ hydroxyurea, $F L=$ first line drug, $S L=$ second line drug.

\subsection{Effect of Patients' Characteristics on Adherence to Medications in the Study Group}

$69.42 \%$ of non-compliant patients were females, $53.8 \%$ housewives and $57.7 \%$ from rural community. $65.4 \%$ of non- compliant patients presented with huge splenomegaly, $66.5 \%$ were fully active, and $61.5 \%$ of them were with chronic phase CML. Out of the non-compliant patients $34.6 \%$, $30.8 \%$, and $26.9 \%$ received HU, TKIs as first line and second line, respectively, as in table 3 .

Table 3. Effect of patients' and disease's characteristics on patients' compliance to treatment.

\begin{tabular}{|c|c|c|c|}
\hline \multirow{2}{*}{$\begin{array}{l}\text { Patients' characteristics, } \\
\mathrm{ttt} \text { and } \mathrm{ttt} \text { side effects }\end{array}$} & \multicolumn{3}{|c|}{ Patients' compliance } \\
\hline & Compliant & Non-compliant & P value \\
\hline \multicolumn{4}{|l|}{ Gender } \\
\hline Male & $11(52.4 \%)$ & $8(42.41 \%)$ & \multirow{3}{*}{0.133} \\
\hline Female & $10(47.6 \%)$ & $18(69.42 \%)$ & \\
\hline \multicolumn{3}{|l|}{ Residence } & \\
\hline Assiut & $14(66.7 \%)$ & $20(76.9 \%)$ & \multirow{4}{*}{0.179} \\
\hline Sohag & $0(0 \%)$ & $3(11.5 \%)$ & \\
\hline Qena & $2(9.5 \%)$ & $2(7.7 \%)$ & \\
\hline Luxor & $1(4.8 \%)$ & $1(3.8 \%)$ & \\
\hline \multicolumn{4}{|l|}{ Social environment } \\
\hline Urban & $12(57.1 \%)$ & $11(42.3 \%)$ & \multirow{2}{*}{0.312} \\
\hline Rural & $9(42.9 \%)$ & $17(57.7 \%)$ & \\
\hline \multicolumn{4}{|l|}{ Occupation } \\
\hline Housewife & $8(38.1 \%)$ & $14(53.8 \%)$ & \multirow{5}{*}{0.685} \\
\hline Employed & $3(14.3 \%)$ & $2(7.7 \%)$ & \\
\hline Farmer & $4(19.0 \%)$ & $5(19.2 \%)$ & \\
\hline Unemployed & $5(23.8 \%)$ & $3(11.5 \%)$ & \\
\hline Student & $1(4.8 \%)$ & $2(7.7 \%)$ & \\
\hline \multicolumn{4}{|l|}{ Clinical characteristics } \\
\hline \multicolumn{4}{|l|}{ Complaint: } \\
\hline Abdominal pain & $14(66.7 \%)$ & $12(46.2 \%)$ & \multirow{4}{*}{0.448} \\
\hline Manifestations of anemia & $6(28.6 \%)$ & $12(46.2 \%)$ & \\
\hline $\begin{array}{l}\text { Manifestations of } \\
\text { pancytopenia }\end{array}$ & $1(4.8 \%)$ & $1(3.8 \%)$ & \\
\hline Atypical chest pain & $0(0 \%)$ & $1(3.8 \%)$ & \\
\hline \multicolumn{4}{|l|}{ ECOG PS } \\
\hline Fully active & $14(66.7 \%)$ & $17(65.4 \%)$ & \multirow{4}{*}{0.238} \\
\hline Restricted strenuous activity & $7(33.3 \%)$ & $6(23.1 \%)$ & \\
\hline Self-care but no work & $0(0 \%)$ & $3(11.5 \%)$ & \\
\hline \multicolumn{3}{|l|}{ Spleen } & \\
\hline Huge splenomegaly & $10(47.6 \%)$ & $17(65.4 \%)$ & \multirow{2}{*}{0.221} \\
\hline Mild to moderate & $11(52.4 \%)$ & $9(34.6 \%)$ & \\
\hline \multicolumn{4}{|l|}{ Type and phase of MPN } \\
\hline Chronic phase CML & $12(57.1 \%)$ & $16(61.5 \%)$ & \multirow{9}{*}{0.339} \\
\hline Accelerated phase CML & $4(19.0 \%)$ & $1(3.8 \%)$ & \\
\hline Blastic phase CML & $1(4.8 \%)$ & $3(11.5 \%)$ & \\
\hline PMF & $1(4.8 \%)$ & $3(11.5 \%)$ & \\
\hline ET & $0(0 \%)$ & $1(3.8 \%)$ & \\
\hline PV & $1(4.8 \%)$ & $0(0 \%)$ & \\
\hline PMF blastic transformation & $1(4.8 \%)$ & $1(3.8 \%)$ & \\
\hline PV fiberotic transformation & $1(4.8 \%)$ & $0(0 \%)$ & \\
\hline Eosinophilic leukemia & $0(0 \%)$ & $1(3.8 \%)$ & \\
\hline \multicolumn{4}{|l|}{ Type of treatment } \\
\hline TKIs first line & $4(19 \%)$ & $8(30.8 \%)$ & \multirow{4}{*}{0.146} \\
\hline TKIs second line & $6(28.5 \%)$ & $7(26.9 \%)$ & \\
\hline HU first or second line & $10(47.6 \%)$ & $9(34.6 \%)$ & \\
\hline Others & $1(4.8)$ & $2(7.6 \%)$ & \\
\hline
\end{tabular}

N.B. ECOG PS= Eastern co-operative group performance status $\mathrm{MPN}=$ Myeloproliferative neoplasms, $\mathrm{CML}=\mathrm{Chronic}$ myeloid leukemia, $\mathrm{PMF}=$ Primary myelofiberosis, $\mathrm{ET}=$ Essential thrombocythemia, $\mathrm{PV}=$ Polycythemia vera, $\mathrm{EL}=$ Eosinophilic leukemia, TKIs=tyrosine kinase inhibitors, HU= Hydroxyurea. 


\subsection{Effect of Adherence to Treatment in the Obtained Therapeutic Response}

We assessed the influence of adherence to TKIs or HU or any given treatment in the study patients on the obtained hematologic response. Results showed that patients who were non-compliant to TKIs were less likely to achieve
CHR. When we considered patients taking HU we found similar results. $42.9 \%$ of compliant patients achieved CHR vs. $23.1 \%$ for those who were non-compliant, PHR in $28.6 \%$ vs. $11.5 \%$, treatment failure in $9.5 \%$ vs. $34.6 \%$, and progression in $9.5 \%$ vs. $26.9 \%, \mathrm{P}=0.068$, as in figure 4 .

\section{Bar Chart}

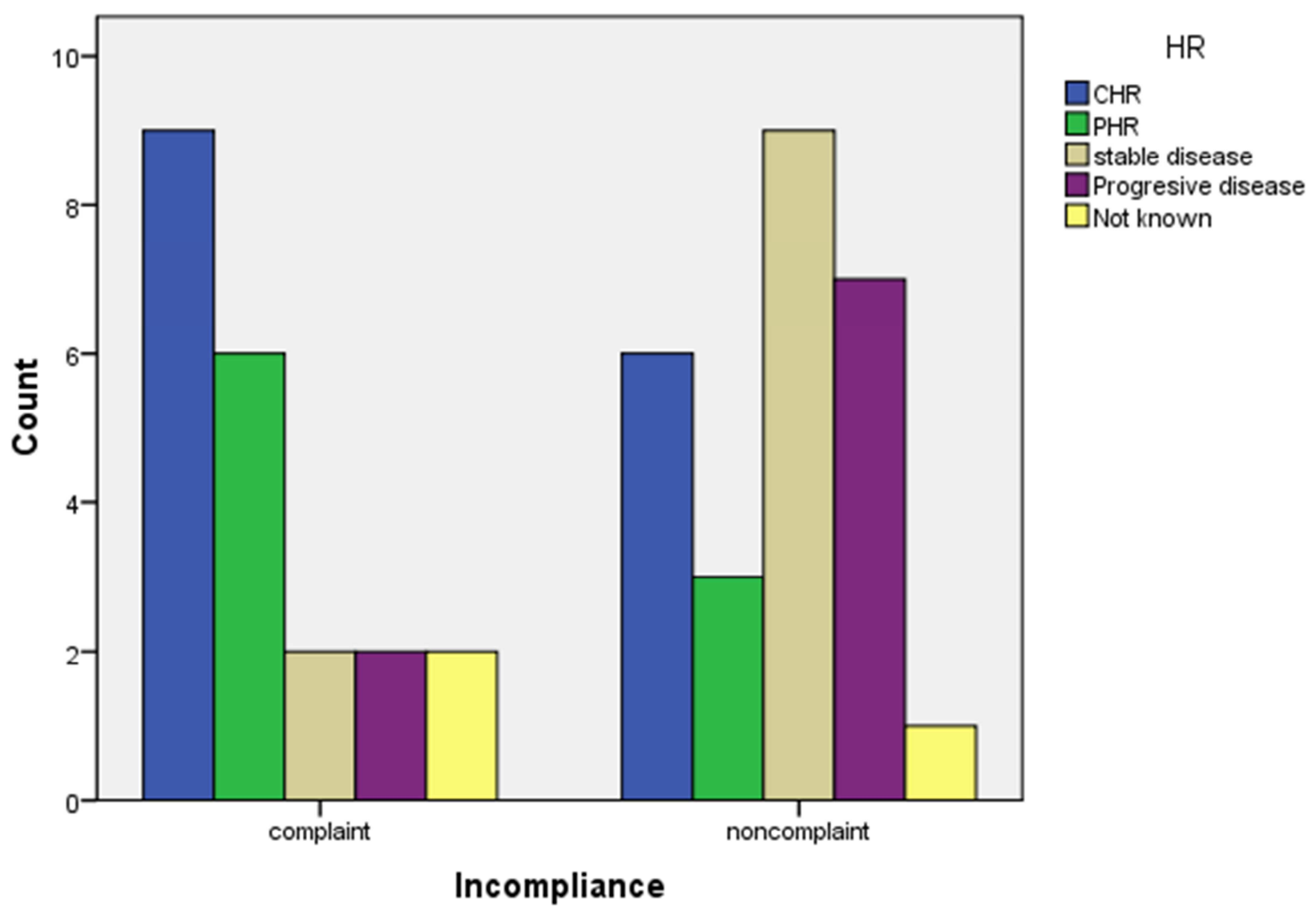

Figure 4. Effect of non-compliance to medications in the obtained hematologic response. N. B. CHR= complete hematologic response. PHR = partial hematologic response.

\subsection{Outcome of Patients Who Were Treated with TKIs Compared to Other Drugs}

Complications of MPNs were present in a considerable proportion of patients. One young age patient with chronic phase CML who were on HU developed lecucytosis $>100$ and thromocytosis that was complicated with priapism, another elderly female developed central nervous system leucostasis that was managed with leucopharesis.

Nearly half of the patients who received TKIs as second line developed blastic transformation, $46.2 \%$ transformed to AML and $7.7 \%$ to ALL. Only one patient of the TKIs first line \& HU groups progressed to ALL \& AML, respectively.

Assessment of response to treatment was mainly based on estimating the hematologic response because a group of patients were from faraway governorates thus long duration of follow up was impractical. The obtained HR showed that the majority who obtained CHR were treated with HU, TKIs as second and first line, in order. $77.8 \%$ \& $22.2 \%$ of those with PHR were treated with HU and TKIs first line respectively. Interestingly, $77.7 \%$ of those who progress to more advanced phases of disease were in the group treated with TKIs as second line drug. Equal percentages of those treated with $\mathrm{HU}$ and TKIs as first line drug developed treatment failure. 3-patients were missed during follow up; accordingly their therapeutic response was unknown. There was no significant difference in PFS \& OS among the study patients who were treated with TKIs compared to other drugs. Table 4, showed outcome of patients with MPNs treated with TKIs compared to other drugs.

$30.8 \% \& 10.5 \%$ of the TKIs second line and HU groups pass their way by the end of the study period. On the contrary the entire TKIs first line group was still living, as in figure 5 . 
Table 4. Outcome of patients with MPNs who were treated with TKIs $(n=25)$ compared to other drugs $(n=22)$.

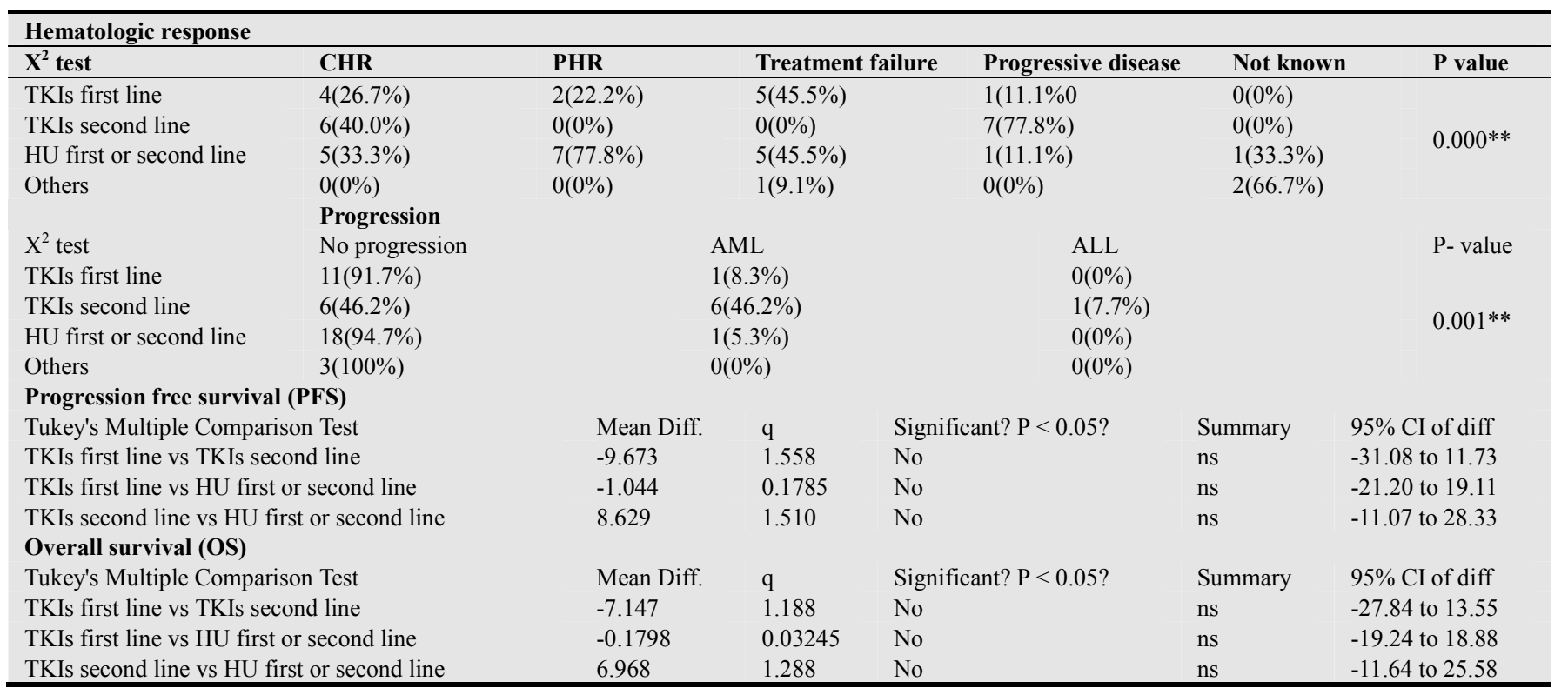

N.B. $\mathrm{CHR}=$ complete hematologic response, $\mathrm{PHR}=$ partial hematologic response, $\mathrm{AML}=$ acute myeloid leukemia, $\mathrm{ALL}=$ acute lymphoblastic leukemia, TKIs=tyrosine kinase inhibitors, $\mathrm{HU}=$ Hydroxyurea

\section{Bar Chart}

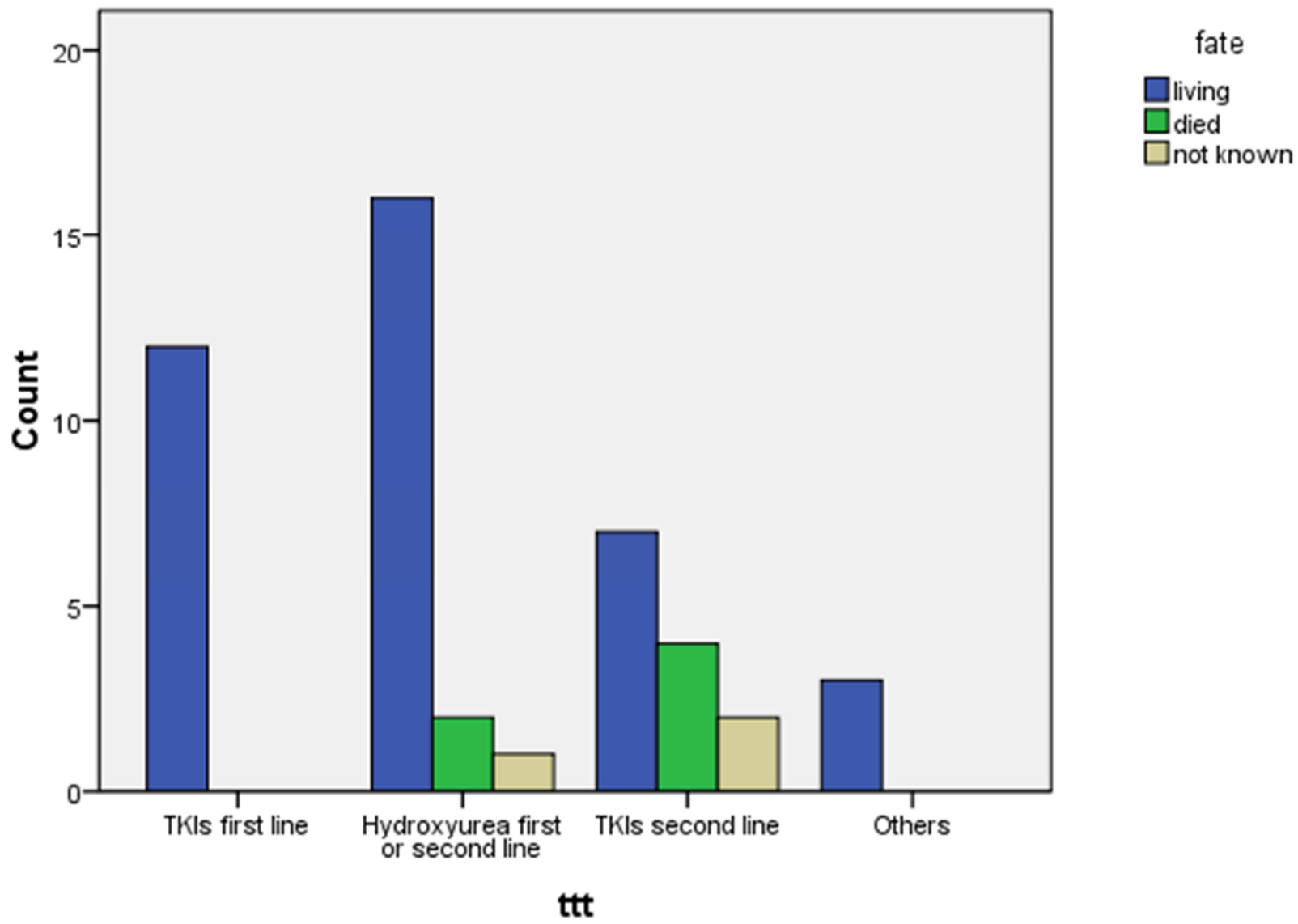

Figure 5. Outcome of patients with MPNs who were treated with TKIs compared to other drugs. $N . B . M P N s=$ myeloproliferative neoplasms, TKIs $=$ tyrosine kinase inhibitors. 


\section{Discussion}

Recently in 2014, the WHO revised its classification of MPNs; all efforts were diverted to the BCR-ABL negative MPNs, [27] furthermore new mutations were discovered in MPNs such as CAlR (calreticulin). [28] This in turn led to the development of new TKIs in MF and PV. [10, 29] All of these denoted that TKIs and their use in MPNs is still a focus for current research. This study was done to assess various aspects of treatment with TKIs in patients with MPNs.

In accordance with recent study in Upper Egypt and contradictory to other studies, this study revealed predominance of female gender and rural residence in patients with MPNs. [30, 31] Furthermore, this study confirmed the findings of others that the most common presenting feature of MPNs is abdominal pain and that CML is the most prevalent MPN. [31, 32]

Although HSCT is the only curative treatment for patients with MPN it was not available for any of our patients. This was, similar to, other studies due to unavailable matched donor and older age of the patients. Furthermore management of MPNs after approval of TKIs was completely changed that HSCT was only reserved to those who developed blastic transformation. [33]

In this study 2- of our patients developed IM failure that was managed by prescribing hydroxyurea as second line treatment. This was mainly due to unavailability and un-affordability of dasatinib or nilotinib as second line drugs. [34]

Adherence to TKIs in this study was markedly lower than that in other studies, and the degree of adherence was found independent risk factor not only for achieving molecular responses but even for HR. [14, 35] This could be explained by the literacy of most of our patients, also due to low socioeconomic status of our patients making TKIs unaffordable. Furthermore, most of our patients were from rural community, faraway from tertiary or even secondary health care centers limiting regular follow up. Unlike other studies, [36] the first most common factor of non-compliance was drug side effects. In Egypt, the Ministry of Health, Government and University Hospitals offer treatments to patients free or with nominal fee, even though cost of therapy was the second most prevalent etiology of non-compliance in the study patients. This could be explained that these services are provided to in-patients, and there are lengthy administrative steps to be provided as outpatient. Furthermore, the chronic course of MPNs makes treatment with the relatively expensive TKIs unaffordable even for those of high socio-economic class.

An interesting finding was that absence of symptoms was the main factor of non-compliance for those treated with TKIs as first line drug. This indicated that the efficacy of TKIs in reducing splenic size, cytoreduction and palliation of constitutional symptoms could be disadvantageous unless patients were alert enough of hazards of non-compliance to TKIs.

This study confirmed that adherence to TKIs is not only crucial for achieving MMR as stated by others, [14, 37] but also in achieving and maintaining CHR. Concomitant with other studies our results showed that non-adherence not only affected therapeutic response to TKIs but to any given drug in the study patients.[38] Non-compliant patients were more susceptible to develop treatment failure and progress to more critical phases of disease. [37] Strikingly, non-compliance to TKIs was more apparent in females, chronic phase CML who were with good ECOG PS.

In this study, drug side effects were more apparent when TKIs were used as second line treatment. Consistent with Masiello et al. this was more prominent in nonhematological side effects such as fluid retention and raised ALT \&AST. [39] When considering hematological side effects isolated cytopenia occurred when TKIs used as first line drugs, while pancytopenia occurred when they were used as second line.

In this study a higher proportion of CML/ PMF patients progressed to blastic phase compared to other studies. This may be due to longer duration of disease besides high rate of non-adherence to medications. [14]

This study proven the efficacy of TKIs in achieving HR, however this was not the case when TKIs were used as second line drugs, this was reflected in very high rate of blastic transformation and mortality of the latter group. These results were conflicting to those of Edesa and his colleague. [40] The appropriate explanation of these conflicting results is much longer duration of disease in patients of this study that ranged from 3-120 months.

This study showed survival advantage of TKIs only when used as first line drug, where there were no detectable deaths in patients treated with TKIs as first line drug while one third and one fifth of the patients who were treated with TKIs as second line drug and HU were died. Nevertheless, there were no significant differences in PFS or OS among the study groups, this could be explained by short duration of follow up in the current study.

Survival advantages of TKIs in patients with MPNs have been proven in previous studies. [40-42] However, this study affirmed that this advantage is gained when TKIs are used as first line and lost or reduced when they are used as second line drugs whether right after HU or even other TKI.

This study showed that myeloblastic transformation is commoner than lymphoblastic in patients with MPNs. An interesting finding was that lymphoblastic transformation occurred only in patients who were treated with TKIs as first or second line drugs. Overall, the majority of blastic transformations were in the group that received TKIs as second line drugs. This could be explained by longer duration of disease in this group with maximum PFS 120month compared with 48-month for other groups. Furthermore, patient adherence to medications progressively reduced with longer duration of follow up. [43, 44] 


\section{Conclusions}

In conclusion, this study showed that although TKIs are effective targeted therapies in patients with MPNs, even though not all patients with MPNs gain benefits of them. This was explained by the unavailability of the newly approved TKIs in most of health care centers. Furthermore their efficacy was reduced by high rates of non-compliance which were mainly due to side effects and high cost of treatment. Tolerability and efficacy of TKIs decreased when they were used as second line drugs. Accordingly, to gain the best results with TKIs in patients with MPNs it is better to use them early in the disease and avoid prescribing cheaper drugs then use TKIs as second line drugs. Furthermore, this paper recommended that health care professionals should include a session of health education for patients with MPN each visit. This session aimed to discuss the value of adherence to TKIs and to teach patients how to overcome any factor of non-compliance. Also hematologists and oncologists have to insist on their patients that absence of symptoms does not mean to stop TKIs. Moreover, they have to take care of patient's socio-economic status when prescribing TKIs.

Also we recommended those who are responsible for synthesizing and marketing TKIs to ensure worldwide distribution and accessibility of drugs in terms of marketing with suitable costs that could be affordable by different socio-economic classes.

Finally, it was concluded that TKIs are still palliative therapies in MPNs and their effect on the natural history of the disease is limited, as stopping drugs could lead to loss of the obtained therapeutic responses. Accordingly their efficacy is limited with non-compliance; hence further research is needed to overcome drawbacks of TKIs and to focus on curative treatments for MPNs.

\section{Acknowledgments}

The author expresses deep thanks and gratitude to medical and paramedical staff at the Hematology and BMT unit, AUH. Special thanks goes to Miss Eman the secretary of the unit for her help during data collection.

\section{References}

[1] Vardiman JW, Thiele J, Arber DA, Brunning RD, Borowitz MJ, Porwit A, et al. The 2008 revision of the World Health Organization (WHO) classification of myeloid neoplasms and acute leukemia: rationale and important changes. Blood 2009; 114: 937-951.

[2] Campbell LJ. Cytogenetics of myeloproliferative neoplasms. Methods Mol Biol. 2011; 730: 89-98.

[3] James C, Ugo V, Casadevall N, Constantinescu SN, Vainchenker W. A JAK2 mutation in myeloproliferative disorders: pathogenesis and therapeutic and scientific prospects. Trends Mol Med. 2005; 11: 546-554.
[4] James C, Ugo V, Le Couédic JP, Staerk J, Delhommeau F, Lacout $\mathrm{C}$, et al. A unique clonal JAK2 mutation leading to constitutive signalling causes polycythaemia vera. Nature. 2005; 434(7037): 1144-1148.

[5] Rollison DE, Howlader N, Smith MT, Strom SS, Merritt WD, Ries LA, et al. Epidemiology of myelodysplastic syndromes and chronic myeloproliferative disorders in the United States, 2001-2004, using data from the NAACCR and SEER programs. Blood. 2008; 112: 45-52.

[6] Espey DK, Wu XC, Swan J, Wiggins C, Jim MA, Ward E, et al. Annual report to the nation on the status of cancer, 19752004 featuring cancer in American Indians and Alaska Natives. Cancer. 2007; 110: 2119-2152

[7] Lahaye T, Riehm B, Berger U, Paschka P, Müller MC, Kreil S, et al. Response and resistance in 300 patients with BCR-ABL-positive leukemia treated with imatinib in a single center: a 4.5-year follow up. Cancer 2005; 103: 1659-1669.

[8] Kantarjian H, Sawyers C, Hochhaus A, Guilhot F, Schiffer C, Gambacorti- Passerini C, et al. International STI571 CML Study Group Hematologic and cytogenetic responses to imatinib mesylate in chronic myelogenous leukemia. N Engl J Med 2002; 346: 645-652.

[9] Redaelli S, Piazza R, Rostagno R, Magistroni V, Perini P, Marega M, et al. Activity of bosutinib, dasatinib, and nilotinib against 18 imatinib-resistant BCR/ABL mutants. J Clin Oncol 2009, 27: 469-471.

[10] Vannucchi A, Kiladjian JJ, Griesshammer M, Masszi T, Durrant S, Passamonti F, et al. Ruxolitinib proves superior to best available therapy in a prospective, randomized, phase 3 study (RESPONSE) in patients with polycythemia vera resistant to or intolerant of hydroxyurea. Haematologica. 2014; 99 (s1): 790; Abstract LB2436

[11] Tefferi A. JAK inhibitors for myeloproliferative neoplasms: clarifying facts from myths. Blood 2012; 119 (12): 2721-2730

[12] Dusing R, Weisser B, Mengden T, Vetter H. Changes in antihypertensive therapy-the role of adverse effects and compliance. Blood Press 1998; 7: 313-5.

[13] Osterberg L, Blaschke T: Adherence to medication. N Engl J Med 2005; 353: 487-497.

[14] Darkow T, Henk HJ, Thomas SK, Feng W, Baladi JF, Goldberg GA, et al. Treatment interruptions and nonadherence with imatinib and associated healthcare costs: A retrospective analysis among managed care patients with chronic myelogenous leukaemia. Pharmacoeconomics 25: 481-496, 2007.

[15] Tefferi A, Vardiman JW. The diagnostic interface between histology and molecular tests in myeloproliferative disorders. Curr Opin Hematol 2007; 14: 115-122.

[16] Barosi G, Ambrosetti A, Finelli C, Grossi A, Leoni P, Liberato $\mathrm{NL}$, et al. The Italian consensus conference on diagnostic criteria for myelofibrosis with myeloid metaplasia. $\mathrm{Br} \mathrm{J}$ Haematol 1999; 104: 730-737.

[17] Tefferi A. Primary myelofibrosis: 2014 update on diagnosis, risk-stratification, and management. Am J Hematol. 2014; 89(9): 915-925. 
[18] Haferlach T, Bacher U, Kern W, Schnittger S, Haferlach C. The diagnosis of BCR/ABL-negative chronic myeloproliferative diseases (CMPD): a comprehensive approach based on morphology, cytogenetics, and molecular markers. Ann Hematol. 2008; 87: 1-10.

[19] Kantarjian HM, Cortes J, Guilhot F, Hochhaus A, Baccarani M, Lokey L. Diagnosis and management of chronic myeloid leukemia: a survey of American and European practice patterns. Cancer 2007; 109: 1365-1375.

[20] Goldman JM, Druker BJ. Chronic myeloid leukemia: current treatment options. Blood 2001; 98: 2039-2042.

[21] Geyer HL, Mesa RA. Therapy for myeloproliferative neoplasms: when, which agent, and how? Blood 2014; 124 (24): 3529-3537.

[22] Kiladjian JJ, Chevret S, Dosquet C, Chomienne C, Rain JD. Treatment of polycythemia vera with hydroxyurea and pipobroman: final results of a randomized trial initiated in 1980. J Clin Oncol. 2011; 29 (29): 3907-3913.

[23] Barbui T, Barosi G, Birgegard G, Cervantes F, Finazzi G, Griesshammer $\mathrm{M}$, et al. Philadelphia-negative classical myeloproliferative neoplasms: critical concepts and management recommendations from European LeukemiaNet. J Clin Oncol. 2011; 29 (6): 761-770.

[24] Jin J, Sklar GE, Min Sen Oh V, Chuen Li S. Factors affecting therapeutic compliance: A review from the patient's perspective. Ther Clin Risk Manag 2008; 4 (1): 269-86.

[25] National Comprehensive Cancer Network. Clinical practice guidelines in oncology, v. 1. 2007; 1-48. Available at: http://www.nccn.org. visited on 24-3-2016.

[26] WMA Declaration of Helsinki - Ethical Principles for Medical Research Involving Human Subjects. 64th WMA General Assembly, Fortaleza, Brazil, October 2013. http://www.wma.net/en/30publications/10policies/b3/. Visited on Jan. 2016.

[27] Tefferi A, Thiele J, Vannucchi AM, Barbui T. An overview on CALR and CSF3R mutations and a proposal for revision of WHO diagnostic criteria for myeloproliferative neoplasms. Leukemia. 2014; 28 (7): 1407-1413.

[28] Nangalia J, Massie CE, Baxter EJ, Gundem G, Wedge DC, Avezov E, et al. Somatic CALR mutations in myeloproliferative neoplasms with nonmutated JAK2. N Engl J Med. 2013; 369 (25): 2391-2405.

[29] Verstovsek S, Kantarjian SH, Mesa RA, Pardanani, CortesFranco J, Thomas DA, et al. Safety and efficacy of INCB018424, a JAK1 and JAK2 inhibitor, in myelofibrosis. N Engl J Med. 2010; 363 (12): 1117-1127.

[30] Khaled SA, Abdel-Aziz NM. Demographic, clinical, and hematologic characteristics of patients with chronic myeloid leukemia in Upper Egypt: association with treatment responses. Egyptian J Haematol 2015; 40: 195-200.

[31] Chen Y, Wang H, Kantarjian H, Cortes J. Trends in chronic myeloid leukemia incidence and survival in the United States from 1975 to 2009. Leuk Lymphoma 2013; 54: 1411.
[32] Siegel R, Ma J, Zou Z, Jemal A. Cancer statistics, 2014. CA Cancer J Clin 2014; 64: 9.

[33] Cherington C, Slack JL, Leis J, Biezen AJ, Bornhäuser M, Di Bartolomeo P, et al. Allogeneic stem cell transplantation for myeloproliferative neoplasm in blast phase. Leuk Res. 2012; 36 (9): 1147-1151.

[34] Kantarjian H, Shah NP, Hochhaus A, Cortes J, Shah S, Ayala $\mathrm{M}$, et al. Dasatinib versus imatinib in newly diagnosed chronic-phase chronic myeloid leukemia. N Engl J Med 2010, 362: $2260-2270$.

[35] Noens L, van Lierde MA, De Bock R, Verhoef G, Zachée P, Berneman Z, et al: Prevalence, determinants, and outcomes of non adherence to imatinib therapy in patients with chronic myeloid leukemia: The ADAGIO study. Blood 113: 54015411, 2009.

[36] Partridge AH, Avorn J, Wang PS, Winer EP. Adherence to therapy with oral antineoplastic agents. J Natl Cancer Inst 94: 652-661, 2002.

[37] Marin D, Bazeos A, Mahon FX, Eliasson L, Milojkovic D, Bua $\mathrm{M}$, et al. Adherence is the critical factor for achieving molecular responses in patients with chronic myeloid leukemia who achieve complete cytogenetic responses on imatinib. J Clin Oncol. 2010. 10; 28 (14): 2381-8.

[38] Ruddy K, Mayer E, Partridge A: Patient adherence and persistence with oral anticancer treatment. CA Cancer J Clin 59: 56-66, 2009.

[39] Masiello D, Gorospe G, Yang AS: The occurrence and management of fluid retention associated with TKI therapy in CML, with a focus on dasatinib. J Hematol Oncol 2009, 2: 46.

[40] Edesa WA, Abdel- Malek RR. Impact of imatinib interruption and duration of prior hydroxyurea on the treatment outcome in patients with chronic myeloid leukemia: Single institution experience. Journal of the Egyptian National Cancer Institute (2015) 27, 69-75.

[41] Alsobhi E, Abrar MB, Abdelaal M, Alsaeed A, Absi A, Zahrani Z, et al. Response to Imatinib therapy in adult patients with chronic myeloid leukemia in Saudi population: single center study. Leuk Lymphoma 2014; 23: 1-15.

[42] Gugliotta G, Castagnetti F, Palandri F, Breccia M, Intermesoli T, Capucci A, et al. Gruppo Italiano Malattie Ematologiche dell'Adulto CML Working Party. Frontline imatinib treatment of chronic myeloid leukemia: no impact of age on outcome, a survey by the GIMEMA CML Working Party. Blood 2011; 117 (21): 5591-9.

[43] Deininger M, O'Brien SG, Guilhot F, Goldman JM, Hochhaus A, Hughes TP, et al. International randomized study of interferon vs STI571 (IRIS) 8-year follow up: sustained survival and low risk for progression or events in patients with newly diagnosed chronic myeloid leukemia in chronic phase treated with imatinib. Blood 2009, 114 (Suppl): 462, (abstract 1126).

[44] Cramer JA, Scheyer RD, Mattson RH. Compliance declines between clinic visits. Arch Intern Med 150: 1509-1510, 1990. 